\title{
Virtual Observatory based identification of AX J194939+2631 as a new cataclysmic variable ${ }^{\star}$ (Research Note)
}

\author{
I. Yu. Zolotukhin ${ }^{1,2,3}$ and I. V. Chilingarian ${ }^{4,2}$ \\ 1 Observatoire de Paris-Meudon, LERMA, UMR 8112, 61 Av. de l'Observatoire, 75014 Paris, France \\ e-mail: iz@sai.msu.ru \\ 2 Sternberg Astronomical Institute, Moscow State University, Universitetskij pr., 13, 119992 Moscow, Russia \\ 3 Observatoire de Paris, VO-Paris Data Centre, 61 Av. de l'Observatoire, 75014 Paris, France \\ 4 Observatoire astronomique de Strasbourg, UMR 7550, Université de Strasbourg/CNRS, 11 rue de l'Université, 67000 Strasbourg, \\ France
}

Received 11 August 2010 / Accepted 11 October 2010

\begin{abstract}
We report the discovery of a new cataclysmic variable (CV) among unidentified objects from the ASCA Galactic Plane Survey made using the Virtual Observatory data mining. First, we identified AX J194939+2631 with IPHAS J194938.39+263149.2, the only prominent $\mathrm{H} \alpha$ emitter among 400 sources in a 1 arcmin field of the IPHAS survey, then secured as a single faint X-ray source found in an archival Chandra dataset. Spectroscopic follow-up with the 3.5-m Calar Alto telescope confirmed its classification as a CV, possibly of magnetic nature. Our analysis suggests that AX J194939+2631 is a medium distance system $(d \approx 0.6 \mathrm{kpc})$ containing a late- $K$ or early- $M$ type dwarf as a secondary component and a partially disrupted accretion disc revealed by the double-peaked $\mathrm{H} \alpha$ line. However, additional deep observations are needed to confirm our tentative classification of this object as an intermediate polar.
\end{abstract}

Key words. novae, cataclysmic variables - X-rays: binaries - X-rays: individuals: AX J194939+2631 -

stars: individual: IPHAS J194938.39+263149.2

\section{Introduction}

Cataclysmic variables are interacting binary systems containing a white dwarf (WD) primary star and a low-mass secondary, usually late-type main sequence star. Moving about the primary star with a typical orbital period of 1 day, the secondary star fills its Roche lobe and experiences mass loss. This matter then spirals down to the non-magnetic WD, forming an accretion disc. These systems are roughly divided into 3 main subtypes: novae (CVs exhibiting thermonuclear bursts on a WD surface resulting in a 6-19 mag increase in their optical luminosities), dwarf novae (CVs where pure disc instability causes quasiperiodic outbursts that temporarily increase optical fluxes by 2-8 mag), and nova-like CVs (non-eruptive CVs characterized by an approximately constant, high-rate mass transfer, a prominent accretion disc, and a high luminosity). Magnetic CVs by definition are those, where magnetic field of the primary companion disrupts the accretion disc, either partially (intermediate polars) or totally (polars). The comprehensive and ultimate overview of CV subclasses and their properties are given in Warner (1995).

Over recent years, numerous authors have undertaken comprehensive CV searches in large publicly available surveys (e.g., Szkody et al. 2002; Witham et al. 2007, 2008; Denisenko \& Sokolovsky 2010). New generation $\mathrm{H} \alpha$ surveys are especially convenient for studies like these. The Isaac Newton Telescope

* Based on observations collected at the German-Spanish Astronomical Center, Calar Alto, jointly operated by the MaxPlanck-Institut für Astronomie Heidelberg and the Instituto de Astrofísica de Andalucía (CSIC).
(INT) Photometric $\mathrm{H} \alpha$ Survey of the northern Galactic plane (IPHAS) covers $\mathrm{a}-5^{\circ}<b<+5^{\circ}$ latitude range providing two broad-band SDSS $r^{\prime}$ and $i^{\prime}$ and a narrow-band $\mathrm{H} \alpha$ magnitudes for about 300 million sources up to $r^{\prime} \sim 20 \mathrm{mag}$. A detailed introduction to the survey is given in Drew et al. (2005).

Using the two-colour diagram $\left(r^{\prime}-i^{\prime}\right)$ versus (vs.) $\left(r^{\prime}-\mathrm{H} \alpha\right)$ as a primary analysis method, Witham et al. (2006) reported a 70 per cent recovery rate of known CVs in IPHAS, because most of them exhibit $\mathrm{H} \alpha$ emission steadily detectable in the IPHAS photometric system. Hence, this method provides a highly efficient selection criterion to distinguish between $\mathrm{CV}$ candidates and field stars.

\section{Identification of AX J194939+2631}

Since we are generally interested in optical/NIR identification of X-ray sources with arcsec-scale error boxes (Zolotukhin et al. 2010; Zolotukhin \& Revnivtsev 2010), we decided to employ the Witham et al. (2006) approach in an attempt to identify CVs among unidentified X-ray sources, with large positional uncertainties, unlikely to be studied by conventional identification techniques.

We used the ASCA Galactic Plane Survey (Sugizaki et al. 2001) carried out in 1996-1999 in a stripe $|l| \lesssim 45^{\circ},|b| \lesssim 0.4^{\circ}$ partially overlapping the IPHAS footprint. This survey, originally intended to study the extended Galactic ridge X-ray emission explained by Revnivtsev et al. (2009), produced 163 point sources brighter than $10^{-12.5} \mathrm{erg} \mathrm{cm}^{-2} \mathrm{~s}^{-1}$ in the $0.7-10 \mathrm{keV}$ range, 107 of which remained unidentified. Since then, some 
authors have attempted to explore unidentified ASCA sources using dedicated observations (see e.g. Kaur et al. 2010), although those studies were hampered by high source density and high probability of random coincidence in 1 arcmin-scale X-ray error boxes of ASCA detections in the Galactic plane.

In our study, we decided to adopt the innovative VO-powered research concept (Chilingarian et al. 2009a) including the three steps: (1) search for candidate object(s) using VO technologies and infrastructure in a semi-automatic way; (2) follow-up observations; (3) interpretation of newly obtained data together with all the information available from the VO. We note that the VObased identification of an X-ray source presented hereafter was made during the tutorial for undergraduate students held at the Sternberg Astronomical Institute in May $2009^{1}$.

As we were to search CVs among unidentified $A S C A$ sources, we first maximized the corresponding probability by preselection of those (1) falling into the IPHAS Initial Data Release (IDR) footprint (33 sources); (2) having hard X-ray spectra (spectral photon index $\Gamma<3$ ); and (3) high estimated column densities $\left(N_{\mathrm{H}}>10^{22} \mathrm{~cm}^{-2}\right)$ indicative of large distances from the Sun. Thus, we obtained a list of six sources that were not expected to be coronal active stars because of the imposed criteria. We then manually explored colour-colour diagrams of IPHAS datasets in 1.3 arcmin circular fields around ASCA coordinates of these sources using VO tools, namely CDS ALADIN (Bonnarel et al. 2000) and TOPCAT (Taylor 2005) for quick-look purposes. We somewhat arbitrarily increased the ASCA error box radius from 1 arcmin uncertainty declared by Sugizaki et al. (2001) to 1.3 arcmin because of our own considerations. On average, there are $\sim 400$ IPHAS sources falling inside such a positional uncertainty.

For one of the sources, AX J194939+2631, we found a single easily detectable prominent $\mathrm{H} \alpha$ emitter in the IPHAS data (see Fig. 1). Despite the presence of other sources with signs of the $\mathrm{H} \alpha$ emission excess, IPHAS J194938.39+263149.2 is the only one in the field exhibiting permanent excess of a high enough confidence level (see typical errors for its magnitude in Fig. 1). From the photometric data, we inferred the $\mathrm{H} \alpha E W$ estimate of 30-60 $\AA$, which is quite typical of a CV. As the candidate falls at the corners of individual IPHAS CCD frames, it was observed three times on Jul. 14, 2005 from 01:04 UT to 02:24 UT, and found to exhibit clear signs of $0.2 \mathrm{mag}$ variability in $i^{\prime}$ and $\mathrm{H} \alpha$ (see Table 1). The source is also present in UKIRT Infrared Deep Sky Survey (UKIDSS) Data Release 4 data (Lawrence et al. 2007). Using only VO resources, we then constructed its optical/NIR spectral energy distribution (SED) shown in Fig. 3. Additional important information about the source was also derived from the USNO-B1.0 catalogue (Monet et al. 2003).

On the basis of these data, we considered IPHAS J194938.39+263149.2 to be a tentative counterpart to AX J194939+2631 and attempted to derive more evidence of their probable association.

\section{Additional data}

\subsection{Chandra $X$-ray data}

After performing the preliminary VO-based identification with the most prominent $\mathrm{H} \alpha$ emitter in the field, we found and analysed yet unpublished publicly available X-ray data of this region

\footnotetext{
1 The complete step-by-step tutorial description is published at the Euro-VO web-site at http: //www . euro-vo.org/pub/fc/workflows.html
}

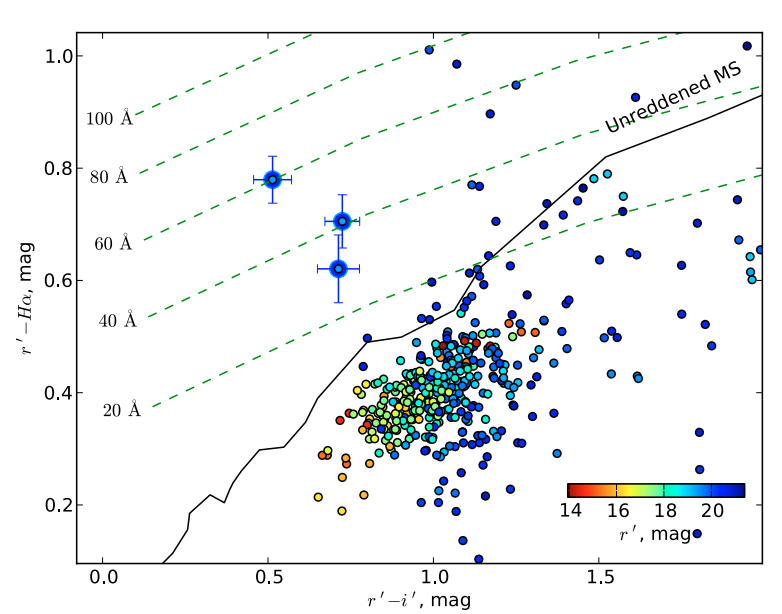

Fig. 1. Colour-colour diagram of the IPHAS detections (not sources) in the 1.3 arcmin field around ASCA coordinates of AX J194939+2631. Objects with $\mathrm{H} \alpha$ excess are located towards the top of the diagram. The $r^{\prime}$ magnitude is colour-coded. Black solid line indicates unreddened main sequence, while green dashed lines are those of constant $\mathrm{H} \alpha E W$ with corresponding values labelled on the left (from Drew et al. 2005). Three detections of IPHAS J194938.39+263149.2 marked with larger circles correspond to the only prominent $\mathrm{H} \alpha$ emitter in the field. Error bars given for it are applicable to other detections as well.

Table 1. Summary of the data on AX J194939+2631, immediately available in the VO after association with IPHAS J194938.39+263149.2 source without additional observations.

\begin{tabular}{|c|c|}
\hline \multicolumn{2}{|r|}{$\overline{A S C A}$} \\
\hline Name & AX J194939+2631 \\
\hline$(l, b)$ & $\left(62.937^{\circ}, 0.203^{\circ}\right)$ \\
\hline Flux, $0.7-10 \mathrm{keV}$ & $0.97 \times 10^{-12} \mathrm{erg} \mathrm{cm}^{-2} \mathrm{~s}^{-1}$ \\
\hline Spectral index & $2.61_{-1.40}^{+2.43}$ \\
\hline Column density & $3.39_{-1.73}^{+1.99} \times 10^{22} \mathrm{~cm}^{-2}$ \\
\hline \multicolumn{2}{|r|}{ IPHAS and UKIDSS } \\
\hline Name & IPHAS J194938.39+263149.2 \\
\hline RA, Dec (J2000) & $\left(297.409912^{\circ}, 26.530325^{\circ}\right)$ \\
\hline$r^{\prime}$ & $19.42 \pm 0.02,19.48 \pm 0.03,19.43 \pm 0.02$ \\
\hline$i^{\prime}$ & $18.69 \pm 0.05,18.76 \pm 0.06,18.91 \pm 0.05$ \\
\hline $\mathrm{H} \alpha$ & $18.71 \pm 0.04,18.86 \pm 0.05,18.65 \pm 0.03$ \\
\hline $\mathrm{H} \alpha E W$ estimate & variable, $30-60 \AA$ \\
\hline$J$ & $17.72 \pm 0.02$ \\
\hline$H$ & $17.40 \pm 0.03$ \\
\hline$K$ & $17.08 \pm 0.07$ \\
\hline \multicolumn{2}{|r|}{ USNO-B1.0 } \\
\hline Name & USNO-B1.0 1165-0456015 \\
\hline$B 1$ & $20.04 \mathrm{mag}$ \\
\hline$B 2$ & $20.20 \mathrm{mag}$ \\
\hline$R 1$ & $20.12 \mathrm{mag}$ \\
\hline$R 2$ & $19.42 \mathrm{mag}$ \\
\hline proper motion, RA & $-8 \pm 1 \mathrm{mas} /$ year \\
\hline proper motion, Dec & $-4 \pm 11$ mas/year \\
\hline \multicolumn{2}{|r|}{ Chandra } \\
\hline Flux, $0.5-10 \mathrm{keV}$ & $1.05 \times 10^{-12} \mathrm{erg} \mathrm{cm}^{-2} \mathrm{~s}^{-1}$ \\
\hline Column density & $5.5 \pm 0.5 \times 10^{22} \mathrm{~cm}^{-2}$ \\
\hline Spectral index & 1.5 \\
\hline
\end{tabular}

Notes. IPHAS magnitudes are given for three consequent epochs (see text). 
obtained with the Chandra satellite on Jan. 8, 2008 in the framework of the Murray (2006) programme (dataset ID 8236).

The Chandra/ACIS X-ray image taken with a $1.74 \mathrm{ksec}$ exposure contains a single faint object within the ASCA error box at the position $\mathrm{RA}=19: 49: 38.39, \mathrm{Dec}=26: 31: 49.1(\mathrm{~J} 2000)$ that within its 0.6 arcsec uncertainty ( 90 per cent confidence) agrees well with the optical position of IPHAS J194938.39+263149.2. The X-ray dataset contains only 116 photons. Translated into $0.5-10 \mathrm{keV}$ flux of $1.05 \times 10^{-12} \mathrm{erg} \mathrm{cm}^{-2} \mathrm{~s}^{-1}$, it does not allow us to characterize its spectrum reliably (see Fig. 4). The best-fit model has a spectral photon index of 1.5 with a column density $N_{\mathrm{H}} \simeq 5.5 \pm 0.5 \times 10^{22} \mathrm{~cm}^{-2}$.

Therefore, the Chandra data allowed us to confirm the association of a prominent $\mathrm{H} \alpha$ emitter with AX J194939+2631, corroborating ASCA estimates of $N_{\mathrm{H}}$ and an X-ray flux. Hence, after careful inspection of VO resources we obtained sufficient information for reliable follow-up studies with dedicated observations (see Table 1). At this stage, it was already clear that the source was either a CV, or a high-mass X-ray binary (HMXB), based on its photometric $\mathrm{H} \alpha E W$ estimate and hard X-ray spectrum.

\subsection{Optical spectroscopic data}

To differentiate between a HMXB and a CV, we performed dedicated optical spectroscopic follow-up observations. The data were collected on Jul. 12, 2009 in the service mode with the TWIN long-slit spectrograph mounted at the $3.5 \mathrm{~m}$ telescope of the German-Spanish Astronomical Centre (Calar Alto Observatory) in the framework of the Director's Discretionary Time proposal "Optical spectroscopy of AX J1949.6+2631" (P.I.: IZ). The total integration time of $2.5 \mathrm{~h}$ was divided into five $1800 \mathrm{sec}$ long exposures. The T08 and T10 gratings were used in the blue and red arms of the TWIN spectrograph simultaneously with the dichroic beam-splitter covering the wavelength bands 3800-5600 $\AA$ and 5550-6900 $\AA$ with the spectral resolving power $R \approx 2500$ and $R \approx 5000$, respectively, for a slit width of 1.2 arcsec. The night-time calibration programme included acquiring bias frames, dome flat fields, He-Ar arc line spectra, and a spectrum of the Feige 110 standard star.

We reduced the data using the generic IFU/longslit data reduction pipeline implemented in IDL following the same steps as those described in Chilingarian et al. (2009b). The only significant difference was the sky subtraction procedure. The TWIN data in our configuration are moderately undersampled in the blue arm and strongly undersampled in the red arm $(F W H M \sim 1$ pix $)$. Therefore, to prevent artifacts originating from the interpolation of undersampled air glow lines, we used the sky subtraction technique proposed by Kelson (2003). We used the entire slit length of 6 arcmin to estimate the oversampled sky spectrum, which we then approximated using the 4th or$\operatorname{der} b$-splines with equidistant nodes every $0.3 \AA$. This procedure resulted in a nearly Poisson sky-subtraction quality.

The source is projected onto a peripheral part of a HII region clearly visible in Fig. 2. Therefore, its spectra are contaminated with narrow nebular emission lines. Since the nebula surface brightness changes along the slit, our sky subtraction technique left significant [positive] residuals at the source position of the spectral lines $\mathrm{H} \alpha$, [NII] $(\lambda=6548,6584 \AA)$ and [SII] $(\lambda=6717,6731 \AA)$. We used two 6 arcsec long segments taken on both sides of the source, interpolated the line residuals in five narrow wavelength regions $( \pm 3 \AA$ ) around these lines, and subtracted them from the source spectrum. The absence of

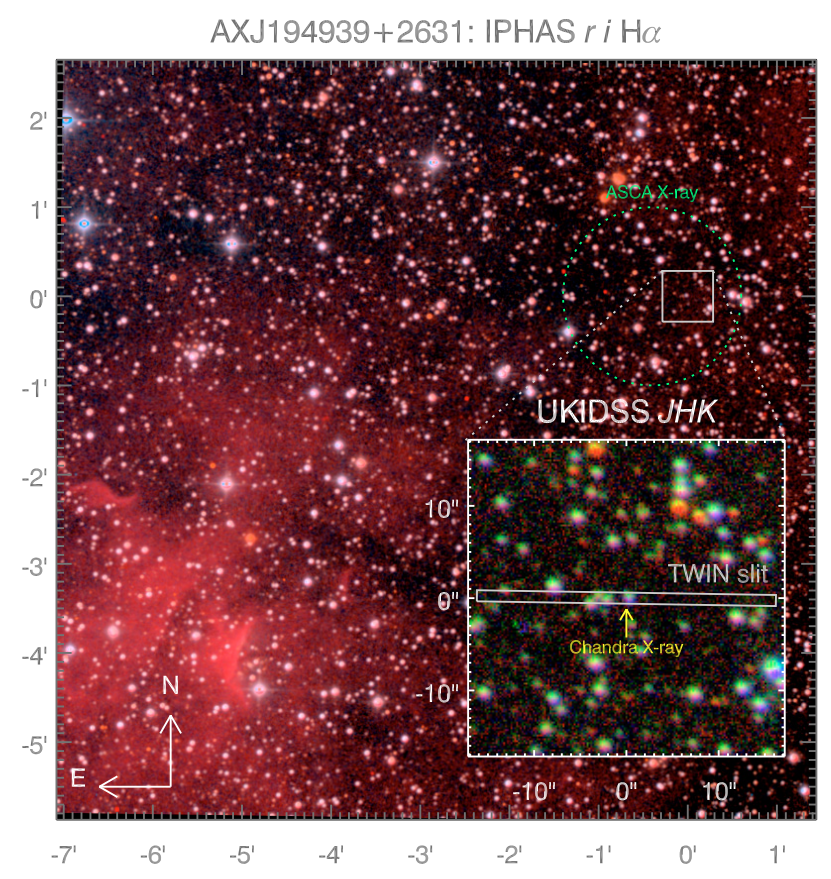

Fig. 2. Data for AX J194939+2631 available in the Virtual Observatory. The background image is a false colour composite made of $r^{\prime}$ and $i^{\prime}$ IPHAS images with $\mathrm{H} \alpha$ added to the red channel using the Lupton et al. (2004) algorithm. The ASCA positional uncertainty is shown as a green circle. A $30 \times 30$ arcsec fragment of the error circle containing the IPHAS J194938.39+263149.2 source as seen in the near-infrared UKIDSS data is displayed in the bottom right inset. The Chandra X-ray source position is shown by an arrow, as well as the slit of the Calar Alto TWIN spectrograph (see Sect. 3.2).

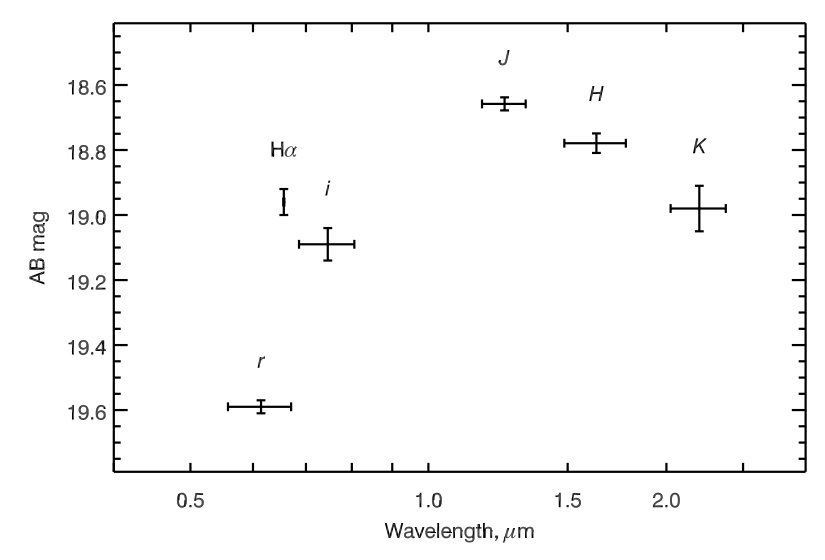

Fig. 3. The reconstructed optical/NIR SED of the source.

any noticeable residuals in the final extracted source spectrum at the position of the four narrow forbidden lines suggests the high reliability of this procedure also in the $\mathrm{H} \alpha$ line.

The sky-subtracted spectra were then linearised using the wavelength solution defined from the automatic identification of the arc line spectra. The systematic errors in the wavelength solution are about $0.07 \AA$ and $0.05 \AA$ in the blue and red arms, respectively.

Interestingly, the residuals of the sky subtraction with the Kelson (2003) technique did not exceed the Poisson noise in the blue arm spectra even in the $\mathrm{H} \beta$ and [OIII] lines, which 


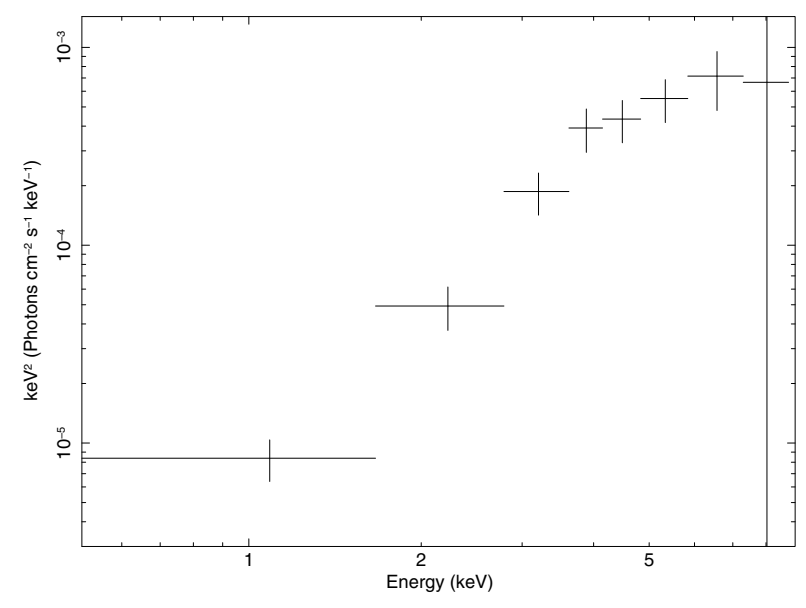

Fig. 4. X-ray spectrum of AX J194939+2631 obtained in a 1.74 ks exposure with Chandra/ACIS detector in a frame of Murray (2006) programme.

Table 2. Equivalent widths of detected emission lines and flux ratios relative to $\mathrm{H} \alpha$.

\begin{tabular}{lrc}
\hline \hline Line & $E W, \AA$ & Line ratio \\
\hline $\mathrm{H} \alpha$ & $-44.7 \pm 2.5$ & 1.0 \\
$\mathrm{H} \beta$ & $-17.3 \pm 3.9$ & $3.5 \pm 1.0$ \\
$\mathrm{H} \gamma$ & $-10.6 \pm 3.3$ & $5.5 \pm 2.0$ \\
$\mathrm{He}$ I 4471 & $-4.0 \pm 3.2$ & $14 \pm 11$ \\
$\mathrm{He}$ I 5876 & $-5.2 \pm 2.5$ & $8.0 \pm 4.2$ \\
$\mathrm{He}$ I 6678 & $-5.8 \pm 1.9$ & $7.3 \pm 2.8$ \\
\hline
\end{tabular}

is indicative of high interstellar extinction and, consequently, a large distance to the HII region.

The coadded, extracted, merged spectrum of IPHAS J194938.39+263149.2, flux-calibrated to an arbitrary constant is displayed in Fig. 5. Several broad emission lines of neutral hydrogen and helium are clearly visible. The HeI line $(\lambda=4471 \AA)$ is detected only marginally, as our measurements are hampered by the low sensitivity in the blue spectral range. Where possible, we computed lines fluxes and equivalent widths by estimating the pseudo-continuum level at the regions beyond $1000 \mathrm{~km} \mathrm{~s}^{-1}$ from the expected line central wavelength assuming zero line-of-sight velocities. The obtained equivalent widths and flux ratios are provided in Table 2.

\section{Discussion}

Both the He emission lines and central $\mathrm{H} \alpha$ absorption, detected at their rest-frame wavelengths, as well as the non-zero proper motion of the object in USNO-B1.0 (at least along one coordinate, see Table 1), clearly indicate that this optical source belongs to the Galaxy. The central absorption in $\mathrm{H} \alpha$ has zero velocity and velocity uncertainty of $\pm 50 \mathrm{~km} \mathrm{~s}^{-1}$. If the source had been distant, at this Galactic longitude $(l=62.9 \mathrm{deg})$ its radial velocity would have a significant non-zero value because of the Galactic differential rotation. The negative sign of its RA proper motion also implies that it resides in the foreground part of the Galaxy, i.e., much closer than $8 \mathrm{kpc}$. Its relatively nearby location automatically rules out the high-mass X-ray binary nature of our object, because its derived X-ray luminosity is too low for this class of sources at $L_{X} \simeq 4 \times 10^{33}(d / 8 \mathrm{kpc})^{2} \mathrm{erg} \mathrm{cm}^{-2}$.

The observed small Balmer decrement is likely indicative of insignificant interstellar reddening. The assumptions of typical conditions in the emitting region $(\mathrm{H} \alpha / \mathrm{H} \beta$ flux ratio of 2.86) and the standard extinction law are translated into $E(B-V) \simeq 0.2$ and $A_{V} \simeq 0.6$. In this case, the mismatch with X-ray data affected by significant line-of-sight absorption can be explained by the difference in the geometry of emitting regions: harder radiation comes from the central source passing through its local absorption material, whereas the optical spectrum originates in the outer parts of the accretion disc, which is subject to much lower intrinsic extinction. The circumstellar nature of the X-ray absorption is supported by the whole line-ofsight Galactic extinction of $A_{V} \simeq 21$ mag (Schlegel et al. 1998) not being enough to account for ASCA and Chandra values of $N_{\mathrm{H}} \sim 3-5 \times 10^{22} \mathrm{~cm}^{-2}$, assuming a standard extinction law with $R=3.1$. The 3D Galaxy extinction map (Marshall et al. 2006) provides an estimate of $A_{V} \simeq 2 \mathrm{mag}$ at a distance of $1.5 \mathrm{kpc}$ in this direction, suggesting that the source resides at $d \simeq 500 \mathrm{pc}$, if we scale the extinction linearly.

The optical/NIR SED of the source (see Fig. 3) closely matches that of an early-M dwarf, with a pronounced blue excess in its $r^{\prime}-i^{\prime}$ colour (Hewett et al. 2006) that occupies the most densely populated region in the colour distribution of known CVs (Witham et al. 2006). Weak barely detected $\mathrm{Mg} b$ absorption and the lack of strong molecular bands and red continuum are consistent with the same classification. Early-M dwarfs are faint objects with a relatively narrow range of absolute magnitudes $\left(M_{K}=8 \ldots 9\right)$. Hence, we estimate the distance to the object in a relatively extinction-free NIR band as $d \simeq 400 \ldots 650 \mathrm{pc}$, which is in a good agreement with the value derived above. The source therefore possesses quite moderate X-ray luminosity in the $0.7-$ $10 \mathrm{keV}$ range, $L_{X} \simeq 2 \times 10^{31} \mathrm{erg} \mathrm{cm}^{-2}$ at $600 \mathrm{pc}$, though it is not certain how the intrinsic extinction can affect the observed value.

We have detected hydrogen emission lines upto $\mathrm{H} \gamma$ in the optical spectrum of the source as well as some HeI lines, both almost unambiguously characteristic of CVs. However, similar features may be present in optical spectra of other object classes such as active late-type stars, symbiotics, and Be stars. Symbiotics exhibit the absorption of a late-type giant and a significantly broader $E W$ of $\mathrm{H} \alpha$ (hundreds of $\AA$ ), which we do not observe here. Be stars can be discerned on the basis of their continua: they exhibit a pure SED of an early-type star, which is clearly not the case for our data. Active late-type stars typically have much narrower $\mathrm{H} \alpha$, known to be smaller than $10 \AA$ (Pettersen \& Hawley 1989), as well as strong molecular absorptions, which we do not detect. We stress that these object types are not known to produce such hard X-ray spectra as shown in Fig. 4. Finally, the HeI $(\lambda=6678 \AA)$ emission line is present in the spectrum, which is not expected for other types of systems (Witham et al. 2007). We therefore clearly classify the source as a CV.

Assuming $(V-r)=0$, we can estimate the intrinsic $F_{\mathrm{X}} / F_{\text {opt }}$ ratio to be around 10 , corrected for intervening Galactic extinction in the optical. This quite high X-ray to optical ratio, as well as the hard spectrum and significant intrinsic extinction in $\mathrm{X}$-rays, rule out the $d$ warf nova classification, suggesting that the source is a nova-like variable, whether magnetic or not (Warner 1995). The double-peaked $\mathrm{H} \alpha$ profile (see Fig. 5, right panel) indicates the presence of an accretion disc, so the source is unlikely to be a polar. However, an intermediate polar with an accretion ring at inclinations $i \sim 60 \ldots 70 \mathrm{deg}$ is able to produce this profile with deep absorption and moderate separation between the peaks. We cannot explain the single-peaked redshifted profiles of other hydrogen emission lines without risk of overinterpreting 

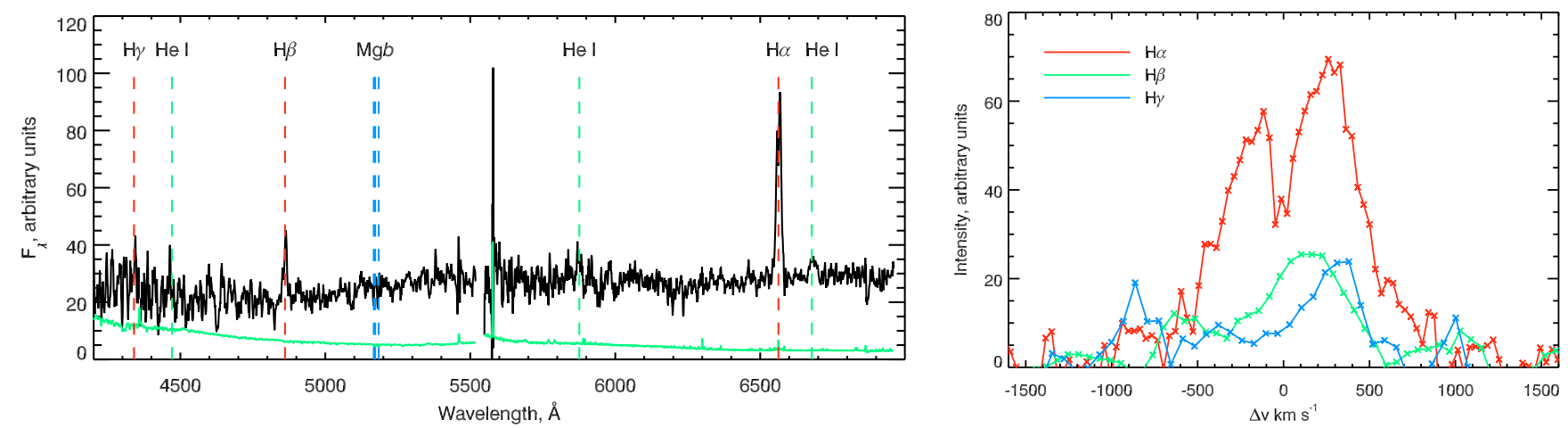

Fig. 5. Left panel: the merged optical spectrum of the AX J194939+2631 counterpart smoothed with a $4 \AA$ box-car for presentation purposes. Right panel: continuum-subtracted profiles of Balmer emission lines. The $\mathrm{H} \beta$ and $\mathrm{H} \gamma$ profiles are smoothed with a $3 \AA$ box-car.

our low signal-to-noise data, but this is possibly because of different geometries of emitting regions (though $\mathrm{H} \alpha$ peaks are also asymmetric, the red one being stronger) and a $2.5 \mathrm{~h}$ long integration during the corresponding phase of a spin wave. At the same time, if the orbital period is comparable to or shorter than $2.5 \mathrm{~h}$, this asymmetry implies that the emission originates predominantly from only one pole (Warner 1995; Hellier 1999).

We notice, however, that the main difficulty with the magnetic CV explanation is the lack of high ionisation lines (HeII, CIII, NIII) in the observed spectrum, although this can be explained by the low signal-to-noise ratio of our optical data. Additional data of higher quality should be obtained to make solid conclusions about the nature of this system. X-ray timing studies to possibly detect periodicities and/or phase-resolved deep spectroscopic observations are required to confirm our tentative classification of this system as an intermediate polar.

Acknowledgements. We thank Calar Alto Observatory for allocation of director's discretionary time to this programme. We would like to thank M. Revnivtsev for his invaluable help with processing of the Chandra dataset. This research has made use of the VizieR catalogue access tool, CDS, Strasbourg, France. I.Z. was supported by Russian state contract No. 02.740.11.0575 and a grant of VO-Paris Data Centre.

\section{References}

Bonnarel, F., Fernique, P., Bienaymé, O., et al. 2000, A\&AS, 143, 33

Chilingarian, I., Cayatte, V., Revaz, Y., et al. 2009a, Science, 326, 1379
Chilingarian, I. V., Novikova, A. P., Cayatte, V., et al. 2009b, A\&A, 504, 389

Denisenko, D. V., \& Sokolovsky, K. V. 2010, [arXiv: 1007.1798]

Drew, J. E., Greimel, R., Irwin, M. J., et al. 2005, MNRAS, 362, 753

Hellier, C. 1999, ApJ, 519, 324

Hewett, P. C., Warren, S. J., Leggett, S. K., \& Hodgkin, S. T. 2006, MNRAS, 367,454

Kaur, R., Wijnands, R., Paul, B., Patruno, A., \& Degenaar, N. 2010, MNRAS, 402, 2388

Kelson, D. D. 2003, PASP, 115, 688

Lawrence, A., Warren, S. J., Almaini, O., et al. 2007, MNRAS, 379, 1599

Lupton, R., Blanton, M. R., Fekete, G., et al. 2004, PASP, 116, 133

Marshall, D. J., Robin, A. C., Reylé, C., Schultheis, M., \& Picaud, S. 2006, A\&A, 453, 635

Monet, D. G., Levine, S. E., Canzian, B., et al. 2003, AJ, 125, 984

Murray, S. 2006, in Chandra Proposal, 2272

Pettersen, B. R., \& Hawley, S. L. 1989, A\&A, 217, 187

Revnivtsev, M., Sazonov, S., Churazov, E., et al. 2009, Nature, 458, 1142

Schlegel, D. J., Finkbeiner, D. P., \& Davis, M. 1998, ApJ, 500, 525

Sugizaki, M., Mitsuda, K., Kaneda, H., et al. 2001, ApJS, 134, 77

Szkody, P., Anderson, S. F., Agüeros, M., et al. 2002, AJ, 123, 430

Taylor, M. B. 2005, in Astronomical Data Analysis Software and Systems XIV, ed. P. Shopbell, M. Britton, \& R. Ebert, ASP Conf. Ser., 347, 29

Warner, B. 1995, Cataclysmic variable stars, Cambridge Astrophysics Series (Cambridge, New York: Cambridge University Press), ed. B. Warner

Witham, A. R., Knigge, C., Gänsicke, B. T., et al. 2006, MNRAS, 369, 581

Witham, A. R., Knigge, C., Aungwerojwit, A., et al. 2007, MNRAS, 382, 1158

Witham, A. R., Knigge, C., Drew, J. E., et al. 2008, MNRAS, 384, 1277

Zolotukhin, I., \& Revnivtsev, M. 2010, MNRAS, accepted [arXiv: 1009. 2454]

Zolotukhin, I. Y., Revnivtsev, M. G., \& Shakura, N. I. 2010, MNRAS, 401, L1 